\title{
ON HOLOMORPHIC FAMILIES OF POINTED RIEMANN SURFACES
}

\author{
BY CLIFFORD J. EARLE ${ }^{1}$ \\ Communicated by F. W. Gehring, June 26, 1972
}

According to a theorem of A. Grothendieck [4] the Teichmüller space of a closed Riemann surface of genus $p \geqq 2$ is the universal parameter space for holomorphic families of marked Riemann surfaces of genus $p$. In this note we offer a corresponding description for every finite-dimensional Teichmüller space $T(p, n)$ and discuss the universal families $\pi: V(p, n) \rightarrow T(p, n)$. Detailed proofs will be given elsewhere.

1. The space $T(p, n)$. Let $X$ be the smooth $\left(C^{\infty}\right)$ oriented closed surface of genus $p \geqq 0$, and let $x_{1}, x_{2}, \ldots$ be a sequence of distinct points on $X$. Set $X_{0}=X, X_{n}=X \backslash\left\{x_{1}, \ldots, x_{n}\right\}, n \geqq 1$. Let $\operatorname{Diff}^{+} X$ be the group of orientation preserving diffeomorphisms of $X$, with the $C^{\infty}$ topology. We define the subgroups

$$
\begin{aligned}
\operatorname{Diff}^{+}(X, n) & =\left\{f \in \operatorname{Diff}^{+} X ; f\left(X_{n}\right)=X_{n}\right\}, \\
G_{n} & =\text { the path component of the identity in } \operatorname{Diff}^{+}(X, n) .
\end{aligned}
$$

Next we form the space $M$ of smooth conformal structures (= complex structures) on $X$, again with $C^{\infty}$ topology. $\operatorname{Diff}^{+} X$ acts on $M$ from the right by pullback. If the inequality

$$
2 p-2+n>0
$$

holds, then the group $G_{n}$ acts freely, continuously, and properly (see [3]) with local sections, and we have a principal $G_{n}$-fibre bundle. The base space $M / G_{n}$ of this bundle is, by definition, the Teichmüller space $T(p, n)$. It is well known that $T(p, n)$ has a natural complex structure and can be imbedded in $C^{d}$ as a bounded open contractible domain of holomorphy $[2], d=3 p-3+n$.

2. $n$-pointed families. Suppose the integers $p, n \geqq 0$ satisfy (1). An $n$ pointed family (of closed Riemann surfaces of genus $p$ ) consists of a pair of complex manifolds $V$ and $B$, a holomorphic map $\pi: V \rightarrow B$, and $n$ holomorphic sections $s_{j}: B \rightarrow V$ such that

(i) $\pi$ is a proper submersion,

\footnotetext{
AMS (MOS) subject classifications (1970). Primary 32G15, $14 \mathrm{H} 15$.

${ }^{1}$ The author is grateful to the Institut Mittag-Leffler for financial support while this research was done.
} 
(ii) $\pi^{-1}(t)$ is diffeomorphic to the closed surface $X$ of genus $p$, for all $t$ in $B$,

(iii) the sections $s_{1}, \ldots, s_{n}$ are disjoint (i.e., $s_{j}(t) \neq s_{k}(t)$ for all $t$ in $B$ if $j \neq k)$.

Given the $n$-pointed family $\pi: V \rightarrow B$, set

$$
V^{\prime}=V \backslash \bigcup_{j=1}^{n} \text { range } s_{j} .
$$

The restriction of $\pi$ maps $V^{\prime}$ onto $B$, and $\pi: V^{\prime} \rightarrow B$ is a smooth fibre bundle with fibre $X_{n}$ and structure group $\operatorname{Diff}^{+}(X, n)$. If the structure group of that bundle is reduced to the subgroup $G_{n}$, we say that the family $\pi: V \rightarrow B$ is marked. In other words, an $n$-pointed family is marked by choosing a homotopy basis on each "punctured fibre" $\pi^{-1}(t) \cap V^{\prime}$ in a manner that depends continuously on $t$.

A map of marked ( $n$-pointed) families is by definition a pair of holomorphic maps $f: V_{1} \rightarrow V_{2}$ and $g: B_{1} \rightarrow B_{2}$ such that $f\left(V_{1}^{\prime}\right)=V_{2}^{\prime}$ and $\left(f^{\prime}, g\right)$ is a map of $G_{n}$-bundles, where $f^{\prime}=f \mid V_{1}^{\prime}$.

THEOREM 1. There is a marked n-pointed family $\pi: V(p, n) \rightarrow T(p, n)$ such that, for every marked $n$-pointed family $\pi_{1}: V_{1} \rightarrow B_{1}$, there is a unique map of marked families

$$
\begin{aligned}
& V_{1} \stackrel{f}{\rightarrow} V(p, n) \\
\pi_{1} & \stackrel{\pi}{\downarrow}{ }^{\prime} \downarrow \\
B_{1} & \stackrel{g}{\rightarrow} T(p, n) .
\end{aligned}
$$

Of course the universal property described in Theorem 1 uniquely determines both $V(p, n)$ and $T(p, n)$ as complex manifolds. For $n=0$, Theorem 1 reduces to Grothendieck's theorem [4]. The general case is proved by the same method. Topologically, $\pi: V(p, n) \rightarrow T(p, n)$ is the $G_{n}$-bundle with fibre $X$ associated to the principal $G_{n}$-bundle $M \rightarrow T(p, n)$ $=M / G_{n}$. The cross-sections of $\pi$ are determined by the points $x_{1}, \ldots, x_{n}$ on $X$ (which are fixed by $G_{n}$ ), and $\pi: V(p, n)^{\prime} \rightarrow T(p, n)$ is the associated bundle with fibre $X_{n}$. The "punctured" fibre space $V(p, n)$ ' is more familiar, and perhaps more natural, than $V(p, n)$. Bers has shown $[1]$ that $T(p, n+1)$ can be interpreted in a natural way as the holomorphic universal covering space of $V(p, n)^{\prime}$.

3. The modular group. Since the group $\operatorname{Diff}^{+}(X, n)$ acts on $M$, and $G_{n}$ is normal in $\operatorname{Diff}^{+}(X, n)$ the quotient group $\Gamma(p, n)$ acts on $T(p, n)$. $\Gamma(p, n)$ is called the (Teichmüller) modular group. This group does not always act effectively on $T(p, n)$; however, it acts also on the fibre space $V(p, n)$ and there it acts very effectively.

THEOREM 2. $\Gamma(p, n)$ acts on $V(p, n)$ and $T(p, n)$ as a group of holomorphic 
automorphisms satisfying

$$
\pi(v \cdot \gamma)=\pi(v) \cdot \gamma \quad \text { for all } v \in V(p, n), \gamma \in \Gamma(p, n) .
$$

Further, if $v \cdot \gamma=v$ for all $v$ in some fixed fibre $\pi^{-1}\left(t_{0}\right)$, then $\gamma=\mathrm{id}$ in $\Gamma(p, n)$.

EXAMPLE. The modular group $\Gamma(2,0)$ has in its center one nontrivial element $\gamma$, of order two. $\gamma$ fixes every point of $T(2,0)$ and therefore maps each fibre $\pi^{-1}(t)$ of $V(2,0)$ onto itself. Each fibre is a closed Riemann surface of genus two, hence hyperelliptic, and $\gamma$ on each fibre is the hyperelliptic involution. Let $\Gamma_{0}=\{\gamma$, id $\}$ be the center of $\Gamma(2,0)$. Then $T(2,0) / \Gamma_{0}$ $=T(2,0) \cong T(0,6)$, and $V(2,0) / \Gamma_{0} \cong V(0,6)$. The six cross-sections of $\pi: V(0,6) \rightarrow T(0,6)$ map $T(0,6)$ onto the six sheets of the branch locus of the map from $V(2,0)$ onto $V(0,6)$. The modular groups $\Gamma(1,1)$ and $\Gamma(1,2)$ also have nontrivial centers which act trivially on $T(1,1)$ and $T(1,2)$, but which act on $V(1,1)$ and $V(1,2)$ by an involution on each fibre.

4. Sections of $\pi: V(p, n) \rightarrow T(p, n)$. John Hubbard has proved [5] that the map $\pi: V(p, 0) \rightarrow T(p, 0)$ has no holomorphic sections if $p \geqq 3$ and exactly six sections if $p=2$. For $n \geqq 1$, the map

$$
\pi: V(p, n) \rightarrow T(p, n)
$$

has $n$ holomorphic sections given, and it makes sense to ask whether $\pi$ has a holomorphic section disjoint from the given ones (i.e., taking its values in $\left.V(p, n)^{\prime}\right)$. We conjecture that no such sections exist unless $p=1$ and $n=1$ or 2 . For the case $p=n=1$, we can prove that only the obvious sections exist. We formulate that fact as

THEOREM 3. Let $U=\{z \in C ; \operatorname{Im}(z)>0\}$ be the upper halfplane. Suppose $f: U \rightarrow C$ is a holomorphic function such that

$$
f(z) \neq m+n z \quad \text { for all } z \in U \text {, all } m, n \in Z .
$$

Then $f(z)=a+b z$, where $a$ and $b$ are real and not both integers.

Our proof of Theorem 3 follows the method of Hubbard in [5]. It would be interesting to have a direct proof.

ADDED IN PROOF. After submitting this paper, the author learned that M. Engber proved a stronger form of Theorem 1 independently in his 1972 Columbia thesis.

\section{REFERENCES}

1. L. Bers, Fibre spaces over Teichmüller spaces (to appear).

2. $\_$Uniformization, moduli, and Kleinian groups, Bull. London Math. Soc. (1972).

3. C. J. Earle and J. Eells, A fibre bundle description of Teichmüller theory, J. Differential Geometry 3 (1969), 19-43. MR 43 \#2737a. 
4. A. Grothendieck, Techniques de construction en géométrie analytique, Séminaire H. Cartan 1960/61, Exposés 7, 17, École Normale Supérieure, Secrétariat mathématique, Paris, 1962. MR 26 \# 3562.

5. J. Hubbard, Sur la non-existence de sections analytiques à la courbe universelle de Teichmüller, C. R. Acad. Sci. Paris Sér A-B 274 (1972), A 978-A 979.

Department of Mathematics, Cornell University, Ithaca, New York 14850

MitTAg-LefFler InStitute, DJuRSholm, SWEDEN 